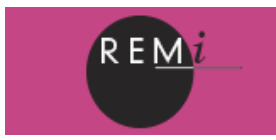

Revue européenne des migrations internationales

vol. 23 - n² | 2007

Numéro ouvert

\title{
Sens et usages de « l'ethnicisation »
}

Le regard majoritaire sur les rapports sociaux ethniques

\section{Hélène Bertheleu}

\section{OpenEdition}

Journals

Édition électronique

URL : https://journals.openedition.org/remi/4167

DOI : 10.4000/remi.4167

ISSN : $1777-5418$

Éditeur

Université de Poitiers

\section{Édition imprimée}

Date de publication : 1 octobre 2007

Pagination : 7-28

ISBN : 978-2-911627-46-6

ISSN : 0765-0752

Référence électronique

Hélène Bertheleu, «Sens et usages de « l'ethnicisation » », Revue européenne des migrations

internationales [En ligne], vol. 23 - n² | 2007, mis en ligne le 01 octobre 2010, consulté le 14 avril 2022.

URL : http://journals.openedition.org/remi/4167 ; DOI : https://doi.org/10.4000/remi.4167

Ce document a été généré automatiquement le 14 avril 2022.

(c) Université de Poitiers 


\title{
Sens et usages de « l'ethnicisation »
}

\author{
Le regard majoritaire sur les rapports sociaux ethniques
}

\author{
Hélène Bertheleu
}

Que signifie exactement ce néologisme florissant, forgé depuis quelques années en France: "l'ethnicisation des relations sociales »? Le succès de cette expression en France n'a d'égal que son absence dans les contextes anglo-saxons, où l'on ne trouve pas de traduction anglaise du terme, de même que dans les contrées francophones comme au Québec. C'est là un phénomène étonnant compte tenu des échanges désormais nombreux entre l'Europe et l'Amérique du Nord, notamment autour des questions de migrations, d'immigration et de relations interethniques, mais aussi en matière de politiques publiques visant la gestion de la diversité culturelle ou la lutte contre les discriminations. Nous proposons d'éclairer ici cet usage situé, son sens "social», son intérêt scientifique et ses implications théoriques et idéologiques. D'apparition récente, puisqu'on ne trouve pas d'occurrence avant 1995, cette notion d'« ethnicisation » semble être, au premier abord, un simple dérivé d'« ethnicité ». Mais les usages, on va le voir, troublent cette idée et nous incitent à clarifier sa généalogie ou ses conditions d'apparition.

2 Contrairement à la notion d'" ethnicisation », le concept d'ethnicité connaît depuis une trentaine d'années des développements théoriques internationaux. La sociologie et l'anthropologie nord-américaines ont, depuis les années 1970, progressivement forgé ce concept pour décrire, dans une approche dynamique et constructiviste, les situations sociales marquées par des relations interethniques ${ }^{1}$. Elles ont fait de l'ethnicité un concept désormais non essentialiste et non substantialiste ${ }^{2}$, permettant d'explorer les dimensions objectives et subjectives des relations entre les groupes ethniques, suivant pour la plupart les premiers travaux wébériens ${ }^{3}$. Loin de se contenter de saisir la seule dimension culturelle de ces relations sociales, ces travaux nous invitent à repérer et étudier l'articulation de ces rapports ethniques avec les rapports de classe et de genre, mais soulignent aussi le potentiel heuristique du concept en mettant en évidence son caractère transversal, soit l'idée que ces rapports ethniques, tout culturels qu'ils nous apparaissent sont aussi, intrinsèquement, économiques et politiques. 
Ce concept d'ethnicité, dont l'intérêt réside dans les nouvelles questions qu'il permet de poser, ne trouva pas en France un terreau de développement théorique comme cela a pu se faire ailleurs ${ }^{4}$, malgré d'excellents travaux en langue française ${ }^{5}$. Pour des raisons historiques et politiques, le concept a longtemps été boudé voire rejeté. Sans revenir sur le contexte qui permet de comprendre ces raisons, rappelons simplement comment, au cours des années 1990, beaucoup d'intellectuels, de chercheurs, de journalistes et, sur le terrain, de travailleurs sociaux, ont été convaincus qu'il fallait résister à la fragmentation, à « l'américanisation » ou à la «balkanisation » de la société française et qu'il fallait dénoncer la montée des particularismes: l'ethnicité fut directement prise dans cette tourmente politique très liée à la question nationale ${ }^{6}$, comme si rejeter le concept participait directement au soutien de la pensée républicaniste et universaliste menacée. Cela justifia que se maintienne jusqu'à aujourd'hui une attitude de retrait scientifique à l'égard des enjeux liés à la différenciation ethnique, comme le suggère entre autres F. Lorcerie (1994) et V. Geisser (1999). Peu saisie scientifiquement, la question reste alors fortement investie par les discours informant la pratique, sociale ou politique (Boubeker, 2003).

Notre propos souhaite au contraire prendre distance avec cette perspective directement orientée par l'idée d'un diagnostic des situations « sensibles", ou celle de l'expertise visant la mise en œuvre ou l'évaluation des politiques publiques. Parce qu'elle est imprégnée de préoccupations d'ordre public et de gestion des populations, cette approche cède à une vision majoritaire ${ }^{7}$ des situations, des relations et des normes, alors même que le défi consiste précisément à saisir simultanément et dans une même dynamique, non pas une mais des visions, montrer à quel point elles sont le reflet des divisions sociales, des classements et des frontières dont elles sont le produit.

5 Peu travaillée scientifiquement, l'ethnicité semble aujourd'hui céder sa place au mot "ethnicisation ». Les travaux français les plus solides sur la question définissent clairement ce processus. L'ethnicisation désigne le processus de la saillance des catégorisations ethniques dans nombre de situations concrètes en France (Poutignat et Streiff-Fénart, 1995 ; De Rudder, 1995 ; Palomares, 2005). Il est souvent utilisé pour décrire et comprendre des conflits impliquant des populations issues de l'immigration dont l'école, le quartier, l'autobus, le guichet de service public ou l'entreprise sont régulièrement le théâtre. Mais en se généralisant, l'expression semble s'être transformée.

\section{Le «franc » succès d'une nouvelle expression}

6 On doit, dans un premier temps, interroger la rapide popularité de ce nouveau vocable. Il semble être, au premier abord, une construction à partir de la notion d'ethnicité. Mais par ailleurs, il appartient aussi à la famille du terme "ethnie » aujourd'hui dénigré et abandonné par les chercheurs, y compris en ethnologie, où l'on use désormais du concept de "groupe ethnique", le plus souvent à l'intérieur du paradigme des frontières ethniques élaboré par Fredrik Barth (1969) et largement repris depuis en anthropologie comme en sociologie. On se trouve donc en France dans une situation étonnante où les ethnies n'ont plus de réalité, où le concept d'ethnicité peine à être utilisé, mais où le processus d'ethnicisation séduit largement tous ceux qui écrivent sur ces questions. Comment expliquer que «l'ethnicisation » ne rencontre pas le même rejet que la notion d'ethnicité? Serait-ce parce que les deux vocables 
renvoient à des visions des choses suffisamment distinctes pour ne pas susciter la même réaction, malgré la proximité sémantique apparente?

7 Une autre hypothèse consiste à se demander si le succès de "l'ethnicisation " ne permet pas justement de mieux laisser de côté le concept d'ethnicité, malgré le travail théorique qui désormais le soutient solidement. Si c'est le cas, il nous faut éclairer les raisons théoriques ou idéologiques qui conduisent des chercheurs, des journalistes, des élus politiques, des chefs de projet urbain, des chefs de grande entreprise, par exemple, à faire ce même choix sémantique, de façon à explorer le caractère heuristique (ou non) de ce néologisme. Les usages d'« ethnicisation » constituent-ils un regard neuf ou inédit sur les relations interethniques ou signent-ils au contraire l'aveu de l'impensé que constitue le concept d'ethnicité en France? L'exploration que nous en faisons ici nous ramène rapidement à la difficulté proprement française à penser sociologiquement les relations interethniques. En effet, comme nous allons le voir, l'expression autorise divers glissements théoriques et idéologiques que le contexte français, tout particulièrement, encourage.

Plusieurs chercheurs rejoignent le questionnement proposé ici lorsqu'ils prennent acte des difficultés à utiliser désormais cette notion sans en expliquer clairement le sens. Introduisant un programme de recherche sur la question, Jean-Paul Payet (2007) souligne la nécessaire clarification des usages sociaux de l'ethnicité : « On se trouve, dès lors que l'on souhaite faire une synthèse des travaux sur le thème de l'ethnicisation des rapports sociaux (a fortiori dans le champ scolaire), devant une difficulté épistémologique, au sens où la catégorie de l'ethnicité est, dans le contexte français, une construction récente. Il convient donc de savoir de quoi parlent les textes, ceux des chercheurs comme ceux des institutionnels, lorsqu'ils emploient la catégorie de l'ethnicité ".

9 C'est ce que nous proposons de faire ici pour révéler, derrière ces usages, les processus sociaux dont la dimension sémantique ou idéologique n'est finalement qu'une facette. Pour réaliser ce travail d'exploration, nous avons sélectionné un corpus d'une quarantaine de textes d'auteurs variés, articles tirés de revues scientifiques, ou relevant de la presse écrite ou électronique, tous relativement récents, écrit entre 2000 et 2006. Les auteurs de ces textes ${ }^{8}$ appartiennent à quatre catégories qui ne se recouvrent pas ou peu dans notre corpus : la première est constituée de chercheurs en sciences humaines (sociologues, psychologues, politologues) de langue française (français ou plus rarement belges) spécialistes ou non de ces questions. La seconde regroupe des textes écrits par des journalistes de presse quotidienne écrite ou des éditorialistes d'hebdomadaires. La troisième catégorie est la plus difficile à circonscrire: elle regroupe des intellectuels ou des personnes engagés, militants, parfois des élus, en tout cas des acteurs de la vie politique, s'exprimant au nom d'un mouvement, d'un parti ou d'un engagement social ou politique. La quatrième, enfin, rassemble des auteurs décrivant directement leurs pratiques professionnelles, dans l'entreprise, le travail social ou la gestion urbaine. Il s'agit d'un travail exploratoire dont l'objectif ne vise ni l'exhaustivité des usages, ni la quantification des occurrences pour l'une ou l'autre des catégories d'auteurs/textes, lesquelles n'ont d'ailleurs été finalisées qu'au cours du recueil des textes eux-mêmes. Il s'agit avant tout de réaliser un repérage des usages les plus fréquents, de noter éventuellement leur diversité ou au contraire leur homogénéité, et tenter de dresser un paysage sémantique valide. Comme on va le voir, les quatre catégories d'auteurs/textes ne s'avèrent pas un élément 
important pour discriminer les différents usages. Malgré la pluralité des implications sémantiques repérées, on assiste plutôt à une seule et même nébuleuse de sens, structurée par divers glissements : d'une pensée scientifique vers un positionnement "social» plus normatif, de ce dernier vers un raisonnement politique, renvoyant parfois à des « prises de parole » sur le sujet, jugé important, ou prenant sens dans une réflexion sur la construction de nouvelles politiques publiques cherchant à prendre davantage en compte les inégalités ethniques.

\section{Les processus de catégorisation mis en exergue}

Dans les divers textes sélectionnés, nous avons facilement repéré la proximité entre l'idée d'ethnicisation et celle de catégorisation'. Lorsqu'un journaliste déplore dans son texte «l'ethnicisation des relations élèves-enseignants" à l'école, lorsqu'un responsable de formation s'inquiète de «l'ethnicisation des relations professionnelles dans le secteur du travail social », ou encore lorsqu'un responsable de Contrat de Ville dénonce « l'ethnicisation des relations dans les quartiers » des périphéries urbaines, on a chaque fois un usage proche de «la saillance des catégorisations ethniques ». Si l'on remonte un peu dans le temps, on trouve des travaux pionniers comme ceux de JeanPaul Payet sur l'école qui décrit les modes de catégorisation ethnique à l'œuvre dans l'espace scolaire et n'hésite pas à qualifier les dynamiques identitaires qu'il observe en termes d'ethnicité, « une dimension de l'identité sociale et de la catégorisation scolaire que les gens mobilisent parfois » dit-il, notamment en cas de conflit (Payet, 1997).

11 Les processus de catégorisation ethnique constituent en effet une part importante des phénomènes explorés par la recherche sociologique, celle qui notamment se penche sur les productions identitaires et les effets subjectifs dévastateurs de la stigmatisation. Les descriptions nous donnent alors à voir la construction d'identités négatives (construction individuelle ou collective d'invisibilité, honte de soi, rage individuelle ou collective, comportement auto-destructeurs, etc.) particulièrement lorsque la catégorisation est fortement asymétrique ou inégale; plus rarement la description d'identités positives, construites sur des bases moins asymétriques et au prix de processus d'identification et de différenciation.

Pourtant, les analyses semblent s'attarder voir s'enliser dans les manifestations multiples de ce processus de catégorisation jusqu'à passer sous silence d'autres processus qui ne relèvent pas, ou pas seulement, de la catégorisation, comme les processus de communalisation (Weber, 1922 ; Juteau, 1999 ; Winter, 2005 ; Billion, 2007) ou encore les rapports majoritaires/minoritaires (Guillaumin, 1972; Pietrantonio, 2001). Ces derniers méritent pourtant toute notre attention parce qu'ils permettent de saisir l'émergence de frontières ethniques inédites ainsi que les actions mises en œuvre par les individus et les groupes pour transformer ou (re)produire ces frontières sociales. En outre, ce cadre théorique permet d'interroger l'effet des statuts majoritaires et minoritaires sur les processus d'individuation vécus par chacun, de part et d'autre des frontières ethniques. En s'appuyant sur les théorisations récentes développées aux États-Unis, au Canada ou encore en Belgique, on s'aperçoit que l'ethnicité n'est jamais seulement le produit de la catégorisation ethnique, que cette dernière fasse l'objet d'une politique publique explicite, comme au Canada, ou qu'elle s'imprime plus implicitement dans les interactions et relations produites au quotidien, comme c'est le cas en France. Le potentiel théorique de l'ethnicité serait ainsi entamé 
par une vision superficielle des relations ethniques, la seule qui soit, sans doute, compatible avec l'occultation proprement française de l'ethnicité.

Il ne s'agit donc pas ici de sous-estimer le poids de la catégorisation ethnique dans les relations sociales ni dans la construction des groupes et des identités en France. Nous souhaitons plutôt pointer l'idée qu'un développement partiel de ces analyses peut nuire à une approche juste et approfondie du phénomène. Elle consisterait d'une part à ne pas réduire les rapports sociaux ethniques à la seule catégorisation ethnique observée dans les interactions sociales et, d'autre part, à ne pas considérer les appartenances ethniques, lorsqu'elles sont affirmées par les minoritaires, comme une simple réaction aux catégorisations dominantes.

\section{L'ethnicisation ou la réduction du potentiel théorique de l'ethnicité}

14 Quand on se penche sur les usages des chercheurs en sciences humaines, plusieurs suggèrent l'impasse que constituerait une "ethnicisation intégrale du social» (sociologue), ou « une lecture complètement ethnicisé de la réalité » (sociologue) ou encore le «danger d'ethniciser les espaces politiques étudiés » (politologue). À quoi peut bien faire référence cette "ethnicisation intégrale du social »? Cela signifie-t-il que la lunette du chercheur peut être complètement obstruée par une vision complètement « ethnicisée » du monde ? Il tomberait ainsi dans l'erreur qui serait de " voir de l'ethnicité partout ", là où il n'y en a peut-être qu'un peu ou pas du tout? Mais s'agit-il de quantifier le poids de l'ethnicité dans une situation?

Dans le même registre, mais sur un mode plus engagé, l'un des textes propose de " résister à l'ethnicisation » (psychologue), l'autre déplore « l'ethnicisation sauvage des interactions, qui conduit à des discriminations » (politologue) tandis que la plupart des textes développent implicitement ou explicitement l'idée que l'ethnicisation consiste "à voiler la réalité des clivages sociaux». De leur côté, les textes écrits par des journalistes convergent généralement vers une telle prudence: "doit-on accepter l'ethnicisation de la castagne "? demande l'un d'eux lorsque ce qu'il considère comme une simple bagarre est interprétée par un confrère comme une agression antisémite. Les discours plus militants abondent dans ce sens, mais vont plus loin, en soulignant par exemple l'incompatibilité entre les idées républicaines et cette « ethnicisation du social ", ou encore l'urgence à se lever contre cette "nouvelle forme d'idéologie dominante" (leader d'un mouvement antiraciste). Un autre texte, tiré cette fois d'un site militant radical, n'hésite pas à se déclarer "contre l'ethnicisation du monde ». Enfin, du côté des élus, l'expression est pour l'instant peu appropriée par ceux issus de partis traditionnels. C'est moins vrai pour les élus ou militant engagés au nom de la "société civile", et l'on retrouve dans leurs textes les mêmes réticences: "l'ethnicisation, c'est la sclérose des relations sociales », déclare l'un d'eux, rédacteur d'un rapport rendu au gouvernement. Beaucoup plus rares, quelques voix s'élèvent pour une prise en compte pragmatique des discriminations ethnistes et racistes, ce qui amène un chef d'entreprise à en parler de façon cette fois positive en estimant que : «l'ethnicisation des entreprises serait une bonne chose ». L'auteur dès lors ne réfère pas à la production du stigmate ni aux catégorisations telles qu'elles peuvent se lire au fil des interactions sociales à la manière dont le montra E. Goffman, mais en appelle à 
une meilleure reconnaissance (ou mesure) de la diversité ethnique au sein de l'entreprise, de façon à repérer in situ les discriminations et tenter d'y pallier.

Alertés par le risque de cette "ethnicisation intégrale du social», nous pourrions adopter l'idée d'un juste milieu théorique entre le piège d'une interprétation trop ethnicisée et la tentation de rejet pur et simple du concept d'ethnicité. Séduisante par son apparente bonne mesure théorique, l'idée manque toutefois de pendant méthodologique : comment s'y prend-on pour bien «mesurer » l'ethnicité, sans exagérer ni sous-estimer son caractère structurant? Si l'on fait l'analogie avec l'épistémologie féministe telle qu'elle s'invite aujourd'hui dans les sciences humaines ${ }^{10}$, dirait-on, là aussi, que le regard sociologique consiste à trouver la juste dose de féminisme au-delà de laquelle le chercheur tomberait dans l'idéologie ? Sans nul doute, la question gagne à être posée autrement. L'idée de ce juste milieu théorique exprime davantage le malaise que suscite encore le concept d'ethnicité en France, qu'un arbitrage scientifiquement fondé. En considérant ainsi que la tâche du chercheur serait de suivre le chemin du milieu (ni trop «d'ethnicisation» qui nuirait à une vision objective de la réalité sociale, ni d'occultation complète des phénomènes ethniques) on cède, et l'on participe, tout en voulant s'en écarter, au mélange des registres politique et scientifique. Certes, le sociologue ne peut ignorer les réticences du lectorat français et ne peut négliger la question plus large de la réception sociale de tout travail de recherche. Mais il semble bien difficile de montrer la fécondité d'un concept si seule la prudence politique fonde les usages scientifiques que l'on peut en avoir. Une telle posture, enfin, ne permet pas d'aller à l'encontre d'une vision de plus en plus teintée d'alarmisme, faisant de l'ethnicité une dimension pathologique des rapports sociaux.

\section{L'ethnicisation comme phénomène pathologique}

17 C'est à Émile Durkheim que nous devons la première réflexion sur la distinction du normal et du pathologique (1889). Il montre que des phénomènes comme le crime ou le suicide, que l'ensemble de la société considère comme des faits sociaux pathologiques, constitue en réalité des phénomènes «normaux » ou réguliers c'est-à-dire propres à toutes les sociétés. Sans pousser plus loin l'analogie avec les conceptions sociologiques de la déviance ou du crime ${ }^{11}$, rappelons ici un principe élémentaire de la sociologie à savoir la nécessaire distinction entre le problème social et la problématique sociologique. Rejetée comme un " problème social » proprement américain puis comme une perspective sociologique inapplicable en France, l'ethnicité deviendrait aujourd'hui, sous la figure du processus d'ethnicisation, un phénomène pathologique touchant désormais notre société, une dimension non plus seulement embarrassante mais menaçante des rapports sociaux, tel un mal ou une gangrène qui toucherait les quartiers déshérités des villes françaises, après les avoir longtemps épargnés.

On retrouve souvent, dans le sillage de la sociologie des mouvements sociaux, cette thèse de l'ethnicisation de la société française dans une société post-industrielle en crise et conséquence d'un modèle d'intégration " en panne " : ce serait là une réaction à l'exclusion sociale et urbaine, le signe d'une société anomique, d'un dysfonctionnement social profond parce qu'il y aurait rupture du processus «normal » d'intégration de la société. On retrouve aussi souvent, dans les médias, cette idée simplifiée selon laquelle la crise de la société industrielle aurait entraîné les phénomènes ou plutôt les "problèmes » sociaux que l'on a rangés sous le vocable « exclusion», cette situation 
provoquant des réactions de refuge ou de défense, c'est-à-dire des replis ou des crispations ethniques: le raisonnement explique ainsi la pente dangereuse vers l'ethnicisation. Les expressions utilisées révèlent bien l'idée de danger, de piège voire de maladie tapie dans l'ombre et prête à resurgir ${ }^{12}$, comme dans l'extrait suivant : « la tentation de l'ethnicité était latente à l'école, et les transformations conjointes de la société et du système scolaire l'ont réveillée " (sociologue). Plus loin : "on assiste de plus en plus à la montée des catégories ethniques dans le système scolaire et à la tendance de plus en plus forte de l'école à transformer des rapports sociaux et scolaires en rapports ethniques ».

\section{La réduction des rapports sociaux ethniques aux rapports de classe}

Le mot "ethnicisation » suggère ainsi de façon directe ou indirecte une dérive, une évolution anormale des relations sociales, un état pathologique de ce que devraient être «normalement» les rapports sociaux, à savoir des rapports de classe. Qu'un individu ou un groupe tente d'exprimer un désaccord ou une opposition (à l'école, dans le quartier, au travail) en s'appuyant sur une identité collective "ethnique», ce support argumentaire sera jugé douteux ou illusoire ${ }^{13}$, et en tout cas dangereux car susceptible de basculer à tout instant vers une explication culturaliste et essentialiste, pire, comme le masque de la pensée néo-raciste.

Dans les approches néo-marxistes comme celle de Hechter (1976), le chercheur s'intéresse surtout au potentiel mobilisateur de l'ethnicité dans le cadre de rapports qui, pour apparaitre « ethniques » au premier abord, serait profondément des rapports de classe. Si l'on peut débattre scientifiquement de cette question ${ }^{14}$, plusieurs pensent aujourd'hui que les rapports sociaux ethniques, tout autant transversaux que les rapports de classe et de genre, s'articulent aux autres formes de rapports sans s'y confondre. Reprenons rapidement l'exemple de l'institution scolaire, dont bien des articles de presse déplorent qu'elle soit ainsi devenue le théâtre de phénomènes d'ethnicisation. Les sociologues ont depuis longtemps souligné le poids de la dimension sociale ou «de classe " au sein du système scolaire notamment à travers les travaux initiés par P. Bourdieu, et on y reconnaît désormais la présence de la dimension ethnique (Lorcerie, 2003). Compte tenu de l'histoire de l'école, de la laïcité française et de la mission égalitaire que la République confia à l'Éducation nationale dès ses débuts, les inégalités de classe, de genre et ethniques ne peuvent être considérées, par les acteurs scolaires que comme des éléments de dysfonctionnement. Mais quel sociologue, dans une perspective descriptive et analytique, peut sincèrement s'étonner ou juger « anormal » de voir les rapports sociaux inégaux qui structurent la société pénétrer de même les espaces scolaires? On sait aujourd'hui que ces inégalités non seulement pénètrent l'espace scolaire mais sont produites en son sein, dans des relations interethniques complexes qui interdisent de mettre en cause un seul des partenaires de ces relations, que ce soient les élèves et leurs familles migrantes, les enseignants et leur pédagogie ou le «système scolaire » dans son organisation même. Les espaces scolaires ne sont pas et n'ont jamais été à l'abri des classements sociaux quels qu'ils soient ; d'autre part, il serait bien difficile d'expliquer pourquoi cette porosité du système scolaire ne vaudrait que pour les rapports de classe ou de genre. Dès les années 1980, une quantité de travaux «interculturels» signalaient l'émergence d'un 
questionnement au sein de l'école tandis qu'étaient engagées des recherches sociologiques, celles de J.-P. Payet notamment, pour décrire de façon fine ces relations interethniques à l'école. Cette perspective est longtemps restée marginale.

21 Aujourd'hui, on redécouvre ces classements de façon partielle et partiale, comme une nouvelle forme de domination qui ne devrait pas exister en France. Pour un peu, on serait prêt à croire que tout cela nous vient des États-Unis, comme tant d'autres choses $!^{15}$ En réalité, les enfants comme leurs parents migrants ont été longtemps 《invisibilisés $»^{16}$ à l'école comme dans tant d'autres sphères de la vie sociale. La nouveauté réside sans doute aujourd'hui dans les prises de parole ou les gestes posés par ceux que le statut de minoritaire reléguait autrefois dans une attitude "polie », de retrait ou de participation sur-normée. Sans revenir sur la question des émeutes de novembre $2005^{17}$, les micro-conflits (expressions d'opposition, de revendication, de protestation ou stratégies de rupture de l'ordre public) qui se sont multipliés ces dernières années ${ }^{18}$ pourraient être interprétés comme une forme de construction relationnelle ou mieux, comme une forme positive de socialisation, tel que le suggère $G$. Simmel (1995, 1ère éd. 1912) lorsqu'il considère que le conflit non seulement est inhérent à la vie sociale mais contribue, d'une certaine manière, à sa production plurielle. En outre, ce langage du conflit permet que se construisent des prises de position, et ce faisant des locuteurs s'accordent mutuellement sur une certaine légitimité à exister et à dialoguer. L'effet le plus fréquent de ce type de situation est que le groupe majoritaire perd le monopole incontesté du discours sur ces questions, il ne peut plus se contenter de considérer ou "prendre en compte", comme il le disait naguère, les inégalités scolaires, ou les injustices sociales qui touchent les groupes minorisés. Une bonne part des micro-conflits peut ainsi être interprétée comme les manifestations d'une nouvelle agentivité des minoritaires qui se montrent, contre toute attente, capables d'agir et non pas seulement de réagir, s'imposent comme inventifs ou créatifs, acteurs au cœur même de ces relations interethniques. Cette interprétation s'éloigne de l'image pétrifiée de la famille pauvre et " déparentalisée » ou de celle du jeune victime de discriminations privilégiées par la presse, sauf lorsque, de façon récurrente, l'image se retourne brutalement, la victime se transformant soudain en émeutier enragé et destructeur ${ }^{19}$.

Loin de nous dégager de cette préhension pesante des situations, le réductionnisme classiste, qui consiste à ne voir dans les relations interethniques qu'une manifestation de plus des rapports de classe, contribue à alimenter un certain sociologisme et une vision simpliste de la domination.

\section{Pour échapper à l'essentialisme?}

L'analyse sociologique des catégorisations ethniques est souvent l'occasion de dénoncer les processus d'essentialisation ou de naturalisation qui les accompagnent. On sait en effet, depuis les travaux de C. Guillaumin, que la catégorisation ethnique ou raciale, tout comme les catégories de sexe, puisent leur force et leur stabilité dans l'idée de nature (Guillaumin, 2002 (1972), 1992). Cet artifice permet de classer durablement les individus dans des groupes si stables qu'on suppose volontiers l'hérédité d'une génération à l'autre, stabilité qui apparaît finalement comme une propriété substantielle de toute forme de catégorisation ${ }^{20}$. Aussi, un des arguments fréquemment développés en France pour rejeter le concept d'ethnicité consiste à dire qu'il 
constituerait une régression théorique, nous ramenant à une vision figée de la culture, à une approche substantialiste et essentialiste des groupes ethniques, à tous ces pièges autour desquels l'anthropologie a longuement travaillé ces dernières décennies. Mais alors pourquoi le terme « ethnicisation » échappe-t-il à ce raisonnement et ne subit-il pas le même rejet?

Une première réponse s'impose : la terminaison en "tion » suggère un processus, une dynamique de fabrication (artefact) ou de construction des catégories ethniques et cela de façon plus claire que le terme ethnicité, dont le sens commun peut en effet laisser croire en une substance ethno-culturelle qui serait attachée à un individu ou un groupe. Nous aurions donc affaire ici à une simple règle de prudence, demandant au chercheur de préférer "ethnicisation» à "ethnicité » pour échapper au risque d'essentialisation. Cette prudence, toutefois, fait fi des travaux sociologiques nord américains qui ont largement débattu de la question. Le contexte n'y était pas le même puisque les sciences humaines d'Amérique du nord devaient, pour leur part, prendre distance avec un usage social et politique large de la notion. Elles sont toutefois parvenues à dépasser les visions primordialistes, pourtant très partagées, qui postulaient une spécificité des attachements ethniques perçus comme particulièrement forts, premiers, irrationnels, non choisis. Dans cette approche, l'identité de groupe s'impose à tous les individus, comme une dimension non choisie, au-delà de la conscience. Certaines, plus culturalistes (Isajiw, 1972), développèrent l'idée de liens culturels non choisis pour finalement aboutir, aujourd'hui, à l'idée de liens socialement et historiquement construits (Juteau, 1999), souvent instrumentalisés (Glazer \& Co, 1975 ; Hechter, 1978), bien que jamais complètement dissociés de productions historiques et culturelles.

Toutefois, en France, le malaise que suscite l'ethnicité reste perceptible dans de nombreux travaux. La crainte de faire référence, malgré soi, à des relations ou des identités essentialisées se traduit par exemple par un usage abondant de guillemets ou d'expressions (on dira par exemple systématiquement: les phénomènes « dits ethniques ») laissant entendre l'imposture que constituerait une lecture ethnique de la situation. Mais en réalité, on peut se demander si la crainte est, non pas celle d'une lecture «ethnique " (c'est-à-dire faisant référence à l'existence de relations interethniques), mais celle d'une lecture «ethniste » ou "culturaliste », c'est-à-dire cédant aux sirènes de la culture-essence telle qu'elle est souvent véhiculée par les porte-parole des groupes minoritaires ou majoritaires eux-mêmes lorsqu'ils sentent leurs références culturelles menacées ou dévalorisées. À chaque fois que ces précautions de guillemets sont prises, l'auteur assumerait alors implicitement l'équivalence entre culture et ethnicité, voire entre essence et ethnicité, ce dont précisément on cherche ici à se distancer. Dans cette confusion, l'ethnicité ne serait plus qu'un (mauvais) mot pour dire "culturel» ou " racial», dans une approche qui n'échapperait pas à ce que A. Bastenier (2004) appelle la « définition culturaliste de la culture ».

Avec le mot "ethnicisation ", le locuteur s'inscrirait dans le sillage d'une approche constructiviste de l'ethnicité, sans toutefois en assumer l'héritage théorique. Règle de prudence ? On peut douter qu'elle ait une réelle raison d'être si elle consiste finalement à rejeter les théorisations sociologiques de l'ethnicité et à couper la réflexion française des débats internationaux sur ces questions. Partout ailleurs, en effet, les recherches se développent pour tenter de comprendre les phénomènes ethniques pour ce qu'ils sont, 
c'est-à-dire des rapports sociaux inégaux historiquement construits, tandis qu'on persiste souvent en France à voir dans la problématique de l'ethnicité une vision essentialiste de la réalité sociale, sans valeur heuristique.

\section{L'ethnicité contre l'intégration nationale}

Derrière ce qui semble être une règle de prudence scientifique, le succès français du terme " ethnicisation » trahit diverses inquiétudes et notamment cette idée de dérive et de relations «pathologisées ", comme si les phénomènes ethniques constituaient en eux-mêmes une menace pour la « société » qui n'est autre ici que la nation ${ }^{21}$.

La pathologisation des phénomènes ethniques correspond en réalité à une définition étroite de la citoyenneté véhiculée par le paradigme de l'intégration, à savoir que l'identité civique, c'est-à-dire "nationale» dans le contexte français, s'imposerait naturellement si les relations sociales n'étaient pas "perturbées" par une forme d'appartenance jugée archaïque et à laquelle personne ne devrait céder, ni les membres du groupe minoritaire, ni les membres du groupe majoritaire, et encore moins les chercheurs. L'« ethnicisation » est perçue et comprise comme un dysfonctionnement social, comme un processus regrettable qui survient lors des moments de crise, qui pervertit en quelque sorte des rapports sociaux qui seraient, en fait, de nature économique et auraient dû s'exprimer plus clairement de façon politique. On considère ainsi à tort que la "couleur» de ces rapports sociaux n'est ethnique que par détérioration et de façon superficielle, tout comme d'ailleurs l'expression publique (quoique faible) de ces groupes minoritaires n'est ethnique (i.e. culturelle ou religieuse) que parce qu'elle ne sait pas ou ne peut pas être politique. Une telle approche, lorsqu'elle est épousée par la sociologie, risque fort de produire cette étrange « sociologie sans acteur » que dénonce A. Boubeker (2003).

L'ethnicisation des relations sociales suscite une inquiétude multiple. Elle renvoie d'abord à la crainte du retour de conceptions racistes de l'Autre (que ravive le souvenir des méfaits du nazisme, régulièrement entretenu en France), ou plus simplement la peur de voir se "fragmenter " la République d'aujourd'hui, s'effriter le «modèle " national dans un contexte européen fragile. Mais l'inquiétude renvoie aussi à l'anticipation de désordres publics, des violences nourries par les inégalités de toutes natures. Face à ces peurs et incertitudes, les élus de tous bords cherchent à rassurer et "construisent" politiquement la question de l'immigration, sur un mode surtout discursif $^{22}$. Ceux qui, chercheurs en sciences humaines, tentent de saisir scientifiquement ${ }^{23}$ leur objet de recherche peinent à construire l'objectivité autour de cette question et à défendre un regard moins normatif, soit plus symétrique.

\section{Des relations asymétriques à saisir de façon symétrique}

En s'inspirant des travaux sur les controverses et notamment du principe de symétrie tel qu'élaboré par la sociologie des sciences (D. Bloor, 1976 ; D. Vinck, 1995 : 105), nous pouvons aller plus loin dans la compréhension des rapports sociaux ethniques. Appliqué à la sociologie des relations interethniques, il s'agit en effet de décrire avec la même grille d'analyse et avec la même objectivité le groupe majoritaire et le groupe 
minoritaire dans leurs interactions : ce que l'un et l'autre disent (ou ne disent pas), ce qu'ils font ou leurs manières de s'abstenir, sans tomber dans le piège de ne décrire que le poids politique de l'un versus les caractéristiques culturelles de l'autre. Trop souvent en effet, les travaux restituent d'un côté, les politiques publiques mises en œuvre, interprétées comme la traduction de la volonté majoritaire de défendre telle grande valeur jugée universelle et de l'autre, les modes de vie et représentations des minoritaires, sans suffisamment montrer la dialectique qui unit ces deux statuts, et comment les pratiques et représentations des uns peuvent être lues comme une réponse aux pratiques et représentations des autres et vice versa. Les unes sont saisies par les sciences politiques ou juridiques, qui explorent et souvent entérinent les univers sociaux de ceux qui ont le pouvoir de dire la norme ${ }^{24}$, les autres sont plus souvent étudiés par la sociologie et l'anthropologie ou cette partie des sciences humaines que l'on appelle outre-atlantique les sublterns studies, où les longues descriptions culturelles voire culturalistes semblent venir combler l'inexistence politique des " communautés ». Il y a bien des tentatives d'inverser le regard, certains proposant une anthropologie du majoritaire (Abélès, 2005 ; Neveu, 2005 ; Pietrantonio, 2004) tandis que d'autres, à l'inverse, s'appliquent à faire une sociologie politique des minoritaires (Breton, 1991 ; Geisser, 1998 ; Lorcerie, 2003 ; Kokoreff, 2004 ; Martucelli, 2001). S'ils montrent les mécanismes sociaux qui entretiennent les «dominations ordinaires", ils relèvent simultanément les multiples formes d'engagement, de protestation, de résistance, d'empowerment, bref des formes d'action (de) minoritaire(s), mais aussi les ethnicités ou communalisations invisibles du majoritaire. La démarche est heuristique précisément parce qu'elle échappe au misérabilisme comme au populisme; et ce n'est pas un hasard si, dans ces quelques travaux, la distance prise avec l'affirmation de la norme, permet de lire les relations interethniques, sans les surinterpréter. Cette approche reste toutefois à contre-courant et sa réception sociale, sujette à polémique.

31 Ainsi, en adoptant une sociologie de l'ethnicité résolument relationnelle, on pourra mieux saisir, d'une part, le vécu et l'agentivitée ${ }^{5}$ des membres des groupes minorisés, appréhendés alors comme des acteurs et non uniquement comme des victimes de ces rapports ethniques; d'autre part, le groupe majoritaire et ses divers moyens d'expression et d'action, parmi lesquels l'État (les administrations, les politiques publiques) appréhendé comme l'acteur ethnique qu'il est parfois et non uniquement comme une instance neutre en matière d'ethnicité. Seule une telle perspective permet d'étudier les groupes majoritaires/minoritaires comme constitutifs l'un de l'autre, comme les artisans de relations qui les lient et de frontières qui les séparent.

L'enjeu est important en France où le discours de l'ethnicisation semble finalement confiner le sociologue dans une approche qui tend à relayer les aveuglements du groupe majoritaire. L'ethnicité n'est pas uniquement le produit de la catégorisation et de la domination. Croire que le majoritaire ${ }^{26}$, par son activité catégorisante, puisse être entièrement à l'origine de l'ethnicité du minoritaire, est en réalité un raisonnement typiquement majoritaire (Juteau, 1999). Les membres du groupe majoritaire, lorsqu'ils ne s'ignorent plus comme partenaires d'un rapport social particulier, se contentent toutefois de l'idée selon laquelle le minoritaire n'apparait et n'existe que dans et par le regard classant du majoritaire. Ainsi, les appartenances ethniques ne sont pas perçues ni toujours étudiées pour ce qu'elles sont, ou pour ce qu'elles produisent (nouvelles formes de communalisation ou d'engagement dans l'espace public par exemple), mais 
pour ce qu'elles disent de la place qu'elles confèrent dans la structure sociale à ceux qui s'en réclament. De nombreux travaux montrent ainsi combien sont aliénantes les catégorisations en usage ou bien, dans des contextes institutionnels, comment sont parfois discriminatoires les institutions du groupe majoritaire. Si ces approches pointent une facette importante des rapports sociaux ethniques, elles taisent une part de la réalité et ce faisant réduisent l'ethnicité à l'équivalent d'un stigmate que les victimes parviennent, dans le meilleur des cas, à "retourner", pour en faire un emblème au cœur d'une identité essentiellement oppositionnelle. L'ethnicité, amputée de toutes les productions symbolico-culturelles qui la constituent dans la plupart des situations, n'apparaît ici que comme la conséquence identitaire de la minorisation, de la discrimination et de la stigmatisation urbaine ${ }^{27}$.

Non seulement les phénomènes ethniques ne sont pas tous contenus dans la discrimination ou, plus largement, dans le rapport social raciste ${ }^{28}$, mais ce serait une erreur significative de les attribuer, en dernière instance, qu'à la seule perception du majoritaire. Tout se passe en fait comme si les phénomènes ethniques, ayant été occultés en France pendant de longues décennies, étaient désormais pris en compte de façon partiale et asymétrique, à travers le regard du groupe majoritaire à l'endroit d'une population qu'il aurait lui-même (et lui seul) marqué du sceau de la différence.

Cette perception asymétrique nie l'agentivité des groupes minoritaires et fait fi de la complexité des phénomènes ethniques tels qu'ils sont étudiés désormais depuis quelques décennies ${ }^{29}$, à savoir comme des frontières ethniques construites de part et d'autre, des frontières qui ont une histoire mais qui, quotidiennement, continuent simultanément de se transformer et de se cristalliser à partir de relations sociales dont les dimensions économiques, culturelles et politiques doivent être chaque fois décrites.

\section{Des relations réciproques constitutives des individus, des groupes et des institutions}

$\mathrm{Au}$ contraire de ce que suggère cette méfiance à l'égard du phénomène pathologique que serait l'ethnicisation, il est crucial de réaffirmer le potentiel heuristique de l'ethnicité qui nous incite à décrire les frontières complexes entre les groupes, frontières construites certes, mais par l'ensemble des groupes en présence, au passé comme au présent; décrire complètement ces frontières, en cessant une fois pour toutes d'opposer l'ethnique au social, comme c'est si souvent le cas. Les rapports ethniques sont bien sûr des rapports sociaux et il est important de rappeler, comme le montre $\mathrm{F}$. Barth, que les frontières ethniques sont intrinsèquement sociales : "les distinctions ethniques ne dépendent pas d'une absence d'interaction et d'acceptation sociale, mais sont tout au contraire les fondations mêmes sur lesquelles sont bâties des systèmes sociaux plus englobants $»^{30}$. Le processus de dichotomisation sociale ou ce que Barth appelle «les frontières ethniques" sont au cœur de notre approche. Si aujourd'hui elles sont socialement pertinentes au point de canaliser la vie sociale et imposer une certaine organisation des relations entre les individus et les groupes qu'elles produisent, c'est parce que, hier, elles ont été construites de part et d'autre quoique de façon asymétrique, et que cette asymétrie continue d'être entretenue (matériellement et symboliquement) par des barrières et des productions historiques, politiques, économiques, culturelles. 

institutionnelle de l'ethnicité ${ }^{31}$, c'est-à-dire la manière dont les institutions (comme l'école, l'État, les administrations, les partis politiques, les médias, etc.) et les politiques publiques participent à la fabrication de l'ethnicité et à ce titre peuvent être comprises comme des acteurs ethniques (Martiniello, 1998 ; Geisser, 1999 ; Bastenier, 2004). Il nous faut aujourd'hui développer des travaux visant à saisir les groupes minoritaires comme des acteurs à part entière de ces frontières ethniques, et non seulement comme l'objet de catégorisations ethniques. A. Bastenier souligne à juste titre que: «Les descriptions des groupes minoritaires comme des acteurs et non des victimes ethniques sont rares et reviennent à montrer le danger de l'ethnicisation de la société (islamisation, développement d'organisations collectives, altérations rampantes de la laïcité au sein même de l'école, etc., risques terroristes) ». Une telle remarque, dans le contexte français, est doublement « incorrecte » politiquement puisqu'elle s'écarte du discours dénonçant la discrimination et qu'elle semble (mais seule une lecture hâtive de ce texte peut le laisser croire) autoriser le basculement vers une explication (à nouveau) culturaliste.

On sait, grâce à M. Weber (1995, $1^{\text {ère }}$ ed. 1922) et à de récentes relectures de ses travaux (Juteau, 1999 ; Winter, 2004), que les constructions ethniques sont porteuses de statuts, qu'elles sont aussi porteuses de sens, et qu'elles participent aux processus de différenciation et de hiérarchisation qui structurent tout ordre social. L'ethnicité, si elle est souvent instrumentalisée économiquement dans les contextes d'entrepreunariat ethnique ou de "niches » économiques ou encore utilisée comme une arme politique pour mobiliser un collectif ou un électorat, ne se réduit pas à un simple prétexte de mobilisation et ne se développe jamais dans un vide culturel et identitaire. Elle suppose des rapports matériels soutenant des constructions symboliques ou idéelles, ce que Juteau appelle, en inversant la formule bien connue de l'ouvrage de Maurice Godelier, la part réelle de l'idéel (1999 : 77).

Quels sont ces rapports matériels et idéels qui permettent à l'ethnicité de se développer ? Ils sont observables dans les conditions de vie de ceux qui produisent et transmettent concrètement le sentiment d'appartenance à un groupe, rebaptisé «communauté ». Ces rapports matériels ne sont pas des contraintes figées qui pèseraient sur les seuls groupes minoritaires. Ils doivent être saisis de façon dynamique, relationnelle, en montrant la manière dont les groupes majoritaire et minoritaire interagissent et se définissent mutuellement, et laissent à leurs membres le soin de transmettre durablement ces frontières ethniques, pourtant plus mouvantes que le prétendent les uns et les autres. D. Juteau décrit cette transmission comme un long processus de socialisation au cours duquel les femmes jouent un rôle central en entretenant matériellement les plus jeunes au sein des structures familiales, et leur transmettant à travers les manières de faire, d'être et de penser, le projet collectif du groupe. De l'éducation gustative ou culinaire aux histoires lues aux enfants avant le coucher, en passant par l'apprentissage des valeurs essentielles à travers les chants traditionnels ou les pratiques domestiques, la vie familiale assure en quelque sorte la «fabrication» ethnique de ces petits d'hommes et de femmes. Car cette éducation transmet aussi, à travers une culture substantielle, la position du groupe ou si l'on préfère la nature asymétrique de ses relations à l'autre (ou aux autres) groupe vis-à-vis duquel il importe de se définir matériellement, de s'opposer symboliquement. Rapport social universel, l'ethnicité est donc inséparable de cette humanisation (pour ne pas 
dire ethnicisation) des individus et des groupes, qui s'épanouit toujours au sein d'une culture particulière, quel que soit son statut. Elle s'inscrit aussi durablement dans les institutions du groupe majoritaire et se donne à lire dans ses politiques publiques.

Les constructions ethniques sont pour tout acteur individuel et collectif une des grilles de lecture possible de la réalité, une des dimensions de son répertoire d'action et de son identité. Si elles sont nécessairement imbriquées dans des rapports de genre et de classe, elles restent néanmoins lisibles et analysables de façon autonome par le chercheur. Les usages situés de "ethnicisation" participent sans aucun doute aux relations interethniques que le chercheur se donne pour objet. Ils sont donc à étudier pour peut-être y lire, comme on l'a fait ici, un usage majoritaire venant combler un malaise théorique tout en entérinant une lecture asymétrique et normative des rapports ethniques.

\section{BIBLIOGRAPHIE}

ABELES, Marc (2005) Identity and Borders : An Anthropological Approach to EU Institutions, 21th Century Studies, Milwaukee, On-Line Working Papers from the University of Wisconsin, http:// uwm.edu

BASTENIER Albert (2004) Qu'est-ce qu'une société ethnique ? Ethnicité et racisme dans les sociétés européennes d'immigration, Paris, PUF, coll. Sociologies d'aujourd'hui, $346 \mathrm{p}$.

BARTH Fredrik (1995) Les groupes ethniques et leurs frontières, in Philippe Poutignat et Jocelyne Streiff-Fenart, Les théories de l'ethnicité, Paris, PUF 1995, pp. 203-214.

BERTHELEU Hélène (2002) Idéologie urbaine et relations interethniques : quelques remarques, in Les Cahiers du CERIEM, n 9, juin 2002, pp. 79-92. www.ceriem.fr

BILLION Pierre (2005) Quartiers en friche, quartiers en chiffres, in Observatoire des Inégalités, www. inegalites. org., nov.

BILLION Pierre (2007) « Lao et Français dans le Haut-Jura : processus réciproques de communalisation ethnique ", in Esprit Critique. Revue Internationale de sociologie et de sciences sociales, dossier « La communauté n'est pas le communautarisme », à paraître.

BLOOR David (1983, $1^{\text {ère }}$ ed. 1976) Sociologie de la logique : les limites de l'épistémologie, Paris, Pandore. BONNAFOUS Simone (1994) Dire et penser l'autre en France et aux États-Unis, in S. ULMO Ed., L'immigration américaine. Exemple ou contre-exemple pour la France?, Paris, L'Harmattan, 204 p., pp. $65-70$.

BOUBEKER Ahmed (2003), Ethnicité, relations interethniques, ou ethnicisation des relations sociales, Les champs de la recherche en France, VEI-Enjeux, n 130, p. 40.

BRETON Raymond (1991) The Governance of Ethnic Communities. Political Structures and Processes in Canada, Wesport : Greenwood Press. 
ELIAS Norbert \& J.-L. SCOTSON (1997) Les relations entre établis et les marginaux : essai théorique, introduction et préface par M. Wieviorka, Les logiques de l'exclusion, Paris Fayard, 278 p., pp. 29-70.

GANS H. J. (1979) Symbolic Ethnicity : The future of Ethnic Groups and Cultures, in America, Ethnic and Racial Studies, Londres, Routledge \& Kegan Paul, (1), pp. 1-20.

GEISSER V. (1999) La mise en scène républicaine de l'ethnicité maghrébine : discours d'État, discours d'acteurs?, in Catherine Neveu Éd., Espace Public et engagement politique. Enjeux et logique de la citoyenneté locale, Paris, L'Harmattan, 307 p., pp. 203-224.

GEISSER Vincent et Shérazade KELFAOUI (1998) Tabous et enjeux autour de l'ethnicité maghrébine dans le système politique français, Revue Européenne des Migrations Internationales, vol. 14 (2), pp. 19-32.

GLAZER N., MOYNIHAN D.P. (eds) Ethnicity. Theory and Experience, Cambridge, Havard University Press, 1975, $531 \mathrm{p}$.

GODELIER Maurice (1978) La part idéelle du réel, L'Homme, vol. 18, pp. 155-187.

HALL Stuart (1992) New Ethnicity, in J. Donald and A. Rattansi (eds), Race, culture and Difference, London, Sage and Open University, 1992, 300 p., pp. 252-259.

HECHTER M. (1976) Ethnicity and Industrialization : on the Proliferation of the Cultural Division of Labor, Ethnicity, 3, pp. 197-201.

ISAJIW Weinfield (1974) Definitions of ethnicity, Ethnicity, vol. 1, New York, Academic Press, pp. 111-124.

JUTEAU Danielle (1999) L'ethnicité et ses frontières, Montréal, Presses Universitaires de Montréal. KYMLICKA Will (1996) Démocratie libérale et droits des cultures minoritaires, in France Gagnon, Marie Mc Andrew et Michel Pagé, Pluralisme, citoyenneté et éducation, Montréal, L'Harmattan, 248 p., pp. 25-51.

LOCHAK Danièle (1989) La doctrine sous Vichy ou les mésaventures du positivisme in Les usages sociaux du droit, CURAPP-PUF, p. 252.

LORCERIE Françoise (2003) L'école et le défi ethnique, ESF Editeur, Collection Actions Sociales/ confrontations.

LORCERIE Françoise (1994) Les sciences sociales au service de l'identité nationale, in Denis Martin Éd., Cartes d'Identités. Comment dit-on «nous » en politique ?, Paris, Presse de la fondation Nationale des Sciences Politiques, pp. 245-281, 304 p.

MARTINIELLO Marco (1995) L'ethnicité dans les sciences sociales contemporaines, Paris, Puf, col. Que sais-je?

MARTINIELLO Marco (1998) Les immigrés et les minorités ethniques dans les institutions politiques : ethnicisation des systèmes politiques européens ou renforcement de la démocratie?, Revue Européenne des Migrations Internationales, vol. 14 (2).

MOORE Damian (2001) Les politiques de développement social urbain et la gestion de l'ethnicité en France et en Grande-Bretagne, Hommes et Migrations, Paris : ADRI, n 1232, pp. 91-97.

MORILLON Anne (2001) Comment peut-on être français... Sélection des nouveaux Français et motivations des candidats à la naturalisation, in Hélène Bertheleu (éd.), Identifications ethniques. Rapports de pouvoir, compromis, territoire, Paris, L'Harmattan, 273 p. 
NEVEU Catherine (2004) Les enjeux d'une approche anthropologique de la citoyenneté, Revue Européenne des Migrations Internationales, vol. 20 (3), pp. 89-101.

PALIDDA Salvatore (1999) La criminalisation des migrants, in Actes de la Recherche en Sciences Sociales, $\mathrm{n}^{\circ} 129$, sept.

PALOMARES Élise (2005) L'ethnicisation des politiques locales et sociales, in Contretemps, Cité(s) en crise. Ségrégations et résidences dans les quartiers populaires, Éditions Textuel, pp. 93-102.

PARSONS Talcott (1975) Some Theoretical Considerations on the Nature and Trends of Change of Ethnicity, in N. Glazer, D.P. Moynihan Eds., Ethnicity. Theory and Experience, Cambridge, Havard University Press, 1975, 531 p., pp. 53-83.

PAYET Jean-Paul (1997) La catégorie ethnique dans l'espace relationnel des collèges de banlieue : entre censure et soulignement, in F. Aubert, M. Tripier, F. Vourc'h, Jeunes issus de l'immigration. De l'école à l'emploi, Paris, L'Harmattan, pp. 207-218.

PAYET Jean-Paul (2002) « L'ethnicité, c'est les autres ». Formes et enjeux de la relation de l'école aux milieux disqualifiés, in Ville-École-Intégration Enjeux, hors-série, n 6, pp. 55-64, décembre.

PAYET Jean-Paul (2007) L'« ethnicisation » des rapports scolaires et la discrimination ethnique au sein de l'école : un bilan des travaux en France et en Europe. Site du Laboratoire CNRS GRS. En ligne. http://recherche.univ-lyon2.fr/grs/index.php?page $=29$ \& notice $=46$

PIETRANTONIO Linda (2005) Égalité et norme : pour une analyse du majoritaire social in Mots. Les langages du politique, $\mathrm{n}^{\circ} 78$, juillet, pp. 117-127.

PIETRANTONIO Linda (2001) Action positive et égalité : une analyse sociologique, Cahiers du GRES, vol, 2, n 1, Éditions du CEETUM - Université Montréal, pp. 56-71.

PIETRANTONIO Linda (2000) Une dissymétrie sociale : rapports sociaux majoritaires/ minoritaires, Bastidiana, 29-30, janvier-juin : 151-176.

REA Andrea (1998) Immigration et racisme en Europe, Bruxelles, Éditions Complexe.

REA Andrea (2006) Les émeutes urbaines : causes institutionnelles et absence de reconnaissance, Déviance et société, vol. 30, 4, pp. 463-475.

RINAUDO Christian (2000) L'ethnicité dans la cité: Jeux et enjeux de la catégorisation ethnique, Paris, L'Harmattan, Logiques sociales.

RINAUDO Christian (1998) L'imputation de caractéristiques ethniques dans l'encadrement de la vie scolaire, Revue Européenne des Migrations Internationales, vol. 14 (3), pp. 27-43.

ROOSENS Ernest S. (1989) Creating Ethnicity. The Process of Ethnogenesis, Newbury Park, Sage Publications.

RUDDER (de) Véronique (1991) Le racisme dans les relations inter-ethniques in L'Homme et la Société, Paris, Anthropos, 4, pp. 75-92.

RUDDER (de) Véronique (1995) Ethnicisation. Pluriel-Recherche. Vocabulaire historique et critique des relations interethniques, cahier $\mathrm{n}^{\circ} 3$.

RUDDER (de) Véronique, POIRET Christian \& VOURC'H François (2000) L'inégalité raciste. L'universalité républicaine à l'épreuve, Paris, PUF (col. Pratiques théoriques), 213 p., pp. 11-20.

SAYAD Abdelmalek (1999) Immigration et pensée d'État, Actes de la Recherches en Sciences Sociales, Paris, Le Seuil, septembre, $n^{\circ} 129$, pp. 5-14. 
SCHNAPPER Dominique (1991) La France de l'intégration. Sociologie de la nation en 1990, Paris

Gallimard, pp. 95-104, 374 p.

SIMON Pierre-Jean (1970) Ethnisme et racisme ou l'École de 1492, Cahiers Internationaux de Sociologie, vol. XLVIII, 1970, Paris : PUF, pp. 119-152.

SIMON Pierre-Jean (1993) Ethnicité, Pluriel Recherche, Vocabulaire historique et critique des Relations interethniques, Cahier n 1, Paris : L’Harmattan, pp. 65-68.

SIMON Pierre-Jean (1997) Différenciation et hiérarchisation sociales Les Cahiers du Cériem, Université Haute Bretagne, Rennes, $\mathrm{n}^{\circ}$ 2, pp. 27-52.

STASIULIS D.K. (1990) Theorizing connections : Gender, Race, Ethnicity and Class In Peter S. LI, Race and Ethnic Relations in Canada.Toronto, Oxford University Press, pp.269-305.

WEBER Max (1995) Economie et société, t. 2, Paris, Plon, Pocket, (1 $1^{\text {ère }}$ éd en all. 1922, $1^{\text {ère }}$ trad. en franç. Plon, 1969).

WILLIAMS Patrick (1982) The invisibility of the Kalderash of Paris : some aspects of the settlement pattern of the Kalderash of the Paris suburbs, Urban Anthropology, XI/3-4 : 315-346.

VINCK Dominique (1995) Sociologie des sciences, Paris, Armand Colin, 292 p.

WINTER Elke (2004) Max Weber et les relations ethniques. Du refus du biologisme racial à l'Etat multinational, Québec, Les Presses de l'Université Laval.

WIRTH Louis (1945) The problem of minority groups in Robert Linton Ed., The Science of Man in the World Crisis, New York, Columbia Press, pp. 347-372.

\section{NOTES}

1. Voir notamment E.C.W. Isajiw (1974), N. Glazer, D.P. Moynihan (1975), Roosens (1989).

2. En langue française, les travaux de la canadienne Danielle Juteau (1999) et du belge Albert Bastenier (2004) sont particulièrement stimulants.

3. Voir le travail récapitulatif en la matière de Elke Winter (2004).

4. Faire figurer la notion dans le nom même d'un laboratoire ou l'intitulé d'un programme de recherche est à cet égard révélateur. En langue française, c'est fréquent en Belgique et au Québec, très rare en France.

5. Voir l'ouvrage de P. Poutignat et J. Streiff-Fenart (1995) et leur traduction du texte fondateur de F. Barth (1969). Voir aussi les travaux de D. Juteau (1999), de V. de Rudder et al. (2000), de P.J. Simon (1970, 1993, 1997), de M. Martiniello (1995) et plus récemment V. Geisser (1998) et A. Boubeker (2003).

6. La dimension nationale est forte et apparaît nettement dans la cristallisation dans cette même décennie du modèle français d'intégration, qui cherchait à qualifier aussi bien les processus passés qu'à affirmer ce qui devait advenir dans un contexte spécifique, français.

7. Au sens sociologique du terme « majoritaire » que propose C. Guillaumin (2002, 1 ère ed. 1972).

8. Nous avons préféré ne pas mentionner ces auteurs de façon à rester centré sur les textes et le sens dont ils sont porteurs. Le statut notamment professionnel de l'auteur est parfois un élément d'analyse pertinent, pas toujours.

9. La catégorisation sociale met en jeu deux groupes d'individus : ceux qui désignent et devront s'entendre conventionnellement pour telle ou telle désignation; ceux qui sont désignés et n'ont généralement pas le choix de l'être ou pas. Selon le cas, l'acceptation de la désignation peut être volontaire ou involontaire, spontanée ou forcée : le jeu objectif des forces contraint à l'accord celui qui ne dispose pas de pouvoir. Voir C. Guillaumin (1972). 
10. Au congrès 2007 de l'Association canadienne française pour l'avancement des sciences, un colloque de sociologie organisé par un consortium de quatre universités canadiennes s'intitulait : "Épistémologie féministe et différenciation sociale ».

11. Terrain " glissant " s'il en est, risquant de nous ramener bien involontairement à une réalité fort contemporaine qu'est l'amalgame entre criminalité et immigration et ce que certains ont appelé la « criminalisation de l'immigration ». Cf. Salvatore Pallida (1999).

12. Cette analyse est partagée par plusieurs chercheurs qui travaillent sur le racisme et déplorent qu'on le lise trop souvent comme une maladie ou un registre mobilisable en temps de crise, plutôt que de saisir les rapports sociaux qui fondent en réalité tout discours raciste. Voir Réa (1998) et Bastenier (2004).

13. L'identité ethnique comme illusion (dangereuse ou simplement naïve) constitue le credo de la plupart des théorisations marxistes des relations ethniques et plus loin, a été partagée par l'ensemble de la sociologie française, fortement marquée par la prépondérance historique de la «question sociale».

14. Danielle Juteau, dans son ouvrage restitue, en français, une partie de ce débat anglophone. On a par exemple la position marxiste de D. Stasiulis (1990) qui réfute l'existence de rapports ethniques ou raciaux et renvoie leur étude à celle des idéologies.

15. La version scientifique de cette croyance a d'ailleurs contribué à alimenter le discours virulent du contre-modèle américain dit $\mathrm{du}$ " communautarisme » ou du «multiculturalisme ", venant au secours, dans les années 1990, de l'affirmation du modèle français d'intégration.

16. Voir le texte de P. Williams (1982). Par ailleurs, A. Sayad (1999) décrit bien l'injonction à la "politesse» (sociale, économique et politique) qui s'imposait aux travailleurs immigrés des années 1950 à 1980, celle-là même qui humilie encore aujourd'hui leurs enfants.

17. A. Réa (2006) fait une analyse intéressante de ces évènements. Il note d'une part l'interprétation strictement socio-économique des émeutes par les sociologues français au détriment d'une interprétation ethnique davantage privilégiée par les sociologues belges, britanniques ou américains, qui n'ignorent pas pour autant les conditions sociales à l'origine des conflits. D'autre part, il remarque combien les sociologues français sous-estiment la dimension politique des émeutes et, par ailleurs, la dimension institutionnelle des relations interethniques, ce qui nous intéresse plus particulièrement ici.

18. En ce qui concerne le champ scolaire, on a surtout des conflits entre parents et enseignants autour du port du hidjab ou à propos de l'orientation scolaire. Dans le champ urbain, les conflits autour des usages de l'espace public sont nombreux sans pour autant relever d'une seule et même explication.

19. L'allusion est rapide et caricaturale, mais nous laissons à d'autres le soin de décrypter le sens de ces stéréotypes souvent repris dans la presse. Voir par exemple pour la période de trouble de novembre 2005, l'analyse de Pierre Billion (2005) prenant le contre-pied de l'interprétation médiatique du jeune de banlieue, issu de l'immigration et enragé, portant atteinte subitement aux biens privés et publics.

20. Une catégorie sociale qui s'avère fluide ou éphémère perd en effet son pouvoir de classement. 21. C'est ce que remarque aussi $C$. Rinaudo: «On ne peut pas réduire l'ethnicité à une simple manifestation pathogène ou réactive face à une société secrétant de l'anomie. Il faut tenter d'échapper à cette problématique de l'intégration et étudier l'ethnicité sous toutes ses facettes » (2000).

22. La question du séjour des étrangers (et notamment des demandeurs d'asile) sur le territoire a fait l'objet de mesures récentes $(2003,2005)$ mais le traitement social et culturel des populations issues de migrations post-coloniales n'est pas assumé en tant que tel puisqu'il entre, de façon non spécifiée, dans le cadre des politiques sociales et urbaines territorialisées.

23. Il ne s'agit pas de céder à un discours scientiste et d'opposer vainement le politique et le scientifique, tant on a conscience que l'un et l'autre s'informent mutuellement. Ils ne procèdent 
pas, toutefois, de la même démarche. Comme le dit bien Éric Fassin, interrogé sur les usages sociaux et politiques de la science: «le métier des sciences sociales, c'est de décrire le monde. C'est une proposition simple, mais si on la prend au sérieux, elle a des effets importants. Cela veut dire qu'on ne peut pas décider a priori que certaines choses n'existent pas ", Vacarme, printemps 2000, site de la Revue :http://www.vacarme.eu.org/article31.html

24. Voir à ce propos le travail de Danièle Lochack (1989) sur l'implication scientifique et politique des juristes pendant le régime de Vichy.

25. Le terme est utilisé dans les sciences cognitives pour désigner l'expérience d'être l'auteur de et de contrôler ses propres actes, plutôt que d'y être soumis à une injonction extérieure. En sociologie, on le retrouve plutôt en Amérique du Nord, sous le terme agency. Pour un usage très proche de celui que nous en avons ici, voir la thèse de L. Pietrantonio (1999) pour qui l'agentivité est le faire social, la capacité à agir, à transformer, à maîtriser le monde autour de soi, s'opposant à la passivité ou à l'impuissance de celui qui est davantage "agit " par les situations ou les individus qui l'entourent.

26. Parler ainsi au singulier du majoritaire peut sembler un abus de langage, mais fait référence à l'idéal-type du majoritaire et non à un individu particulier. Il est important de rappeler que les membres du groupe majoritaire n'occupent pas tous la même position et donc n'ont pas tous le même pouvoir de nommer, désigner, catégoriser. On sait aujourd'hui la force que peut avoir une politique publique en la matière (pensons aux catégorisations produites en cascade par le RMI, par exemple) mais il ne faut pas non plus négliger des catégories d'usage historiquement légitimées comme celle d' "immigré » en France, catégories qui ont été véhiculées dans le monde économique (et notamment par le patronat qui recrutait à l'étranger après-guerre) avant de se diffuser dans le reste de la société.

27. Cette approche est confirmée par l'histoire récente de la sociologie urbaine française. En revenant un peu sur les années 1980 et 1990, on remarque aisément les «liaisons dangereuses » entre les spécialistes de l'urbain et les tenants d'une approche sécuritaire de l'immigration cf. $\mathrm{H}$. Bertheleu, (2002).

28. C'est plutôt l'inverse qui est affirmé par la plupart des spécialistes : le racisme est un cas particulier des relations interethniques.

29. Cf. revue Ethnicity, depuis 1976.

30. F. Barth, "Les groupes ethniques et leurs frontières ", in P. Poutignat et J. Streiff-Fenart (1995), p. 212.

31. Les travaux de V. de Rudder et alii. (2000), de F. Lorcerie (2003) et V. Geisser (1999) y ont contribué récemment en France.

\section{RÉSUMÉS}

Les sociologues qui œuvrent aujourd'hui dans le champ des relations interethniques ont fait de l'ethnicité un concept dynamique désormais non essentialiste. Si le potentiel heuristique du concept tient dans l'idée que ces rapports ethniques, du fait de leur caractère transversal, sont des rapports intrinsèquement économiques, politiques et culturels, on tend encore trop souvent à les considérer en France comme des relations culturelles ou pseudo-culturelles se développant «à côté » ou masquant des rapports économiques et politiques. Dans le contexte français, l'expression " ethnicisation des relations sociales » est souvent utilisée. Au delà du constat de la saillance des catégorisations ethniques, cette expression marque une volonté de distance avec 
l'approche essentialiste ; mais elle renvoie souvent à une explication en soi alors qu'elle constitue précisément le phénomène à étudier. Certains usages, qu'ils émanent de chercheurs, de journalistes ou encore d'élus, suggèrent clairement qu'il y a là une forme pathologique ou pervertie de relations sociales, un phénomène qu'on déplore et contre lequel il convient de lutter. En questionnant la popularité de cette expression en France et son absence ailleurs, on s'aperçoit qu'elle contribue à occulter les relations interethniques telles qu'elles sont à l'œuvre actuellement en Europe. Le succès de l'« ethnicisation » serait finalement l'aveu de l'impensé de l'ethnicité.

Meanings and Uses of Ethnicisation. Dominant Views on Ethnic Relations. Contemporary works of researchers who have contributed to the sociology of ethnic relations have lead one to consider ethnicity as a dynamic concept free from essentialist and substantialist meanings. The idea that, due to their transversal pattern, ethnic relations are intrinsically economic, political, and cultural relations, encapsulates the potential of the concept to raise unprecedented questions. Yet, in France, ethnic relations are frequently mistaken for cultural or pseudo-cultural relations developing "aside", or combining with, or even masking economic and political relations.

In the French context, and despite the fact that the concept of ethnicity is still struggling to meet an adequate theoretical framework, the phrase "ethnicisation of social relations" is now in undisputed use when it comes to refer to the instrumental process through which ethnic categorizations are made salient. Worth noticing, however, is also the fact that, too often, the mere observation of ethnicisation comes as a substitute explanation in lieu of the actual study of the phenomenon. Specific works clearly suggest that there lies a pathological, perverted pattern of relations that only veils or tones down relations which are conceptualized as standard political and/or economic relations, and therefore studied as such.

Questioning the prevalent use of the phrase "ethnicisation of social relations" in France -and its absence elsewhere-this article examines how such a phrase has contributed to elude ethnic relations that are at stake in contemporary Europe. In contrast, an increasing popular use of the word ethnicisation would eventually yield to acknowledge unthought-of dimensions of ethnicity.

Significados y usos de la "etnicisation". Puntos de vista mayoritarios sobre las relaciones étnicas. Los sociólogos que actualmente ejercen en el campo de las relaciones interétnicas han hecho de la etnicidad un concepto dinámico libre de esencialismos. La idea según la cual, debido a su carácter transversal, las relaciones étnicas son intrínsecamente económicas, políticas y culturales, manifiesta el potencial heurístico del concepto. Sin embargo, en Francia, se tiende todavía a considerar las relaciones interétnicas como relaciones culturales o seudo-culturales que se desarrollan « al margen » llegando incluso a ocultar relaciones económicas y políticas.

En el contexto francés, se utiliza a menudo la expresión "etnicisation de las relaciones sociales". Más allá de la cuestión de la relevancia de las categorizaciones, esta expresión marca una clara voluntad de distancia respecto a la visión esencialista aunque, a menudo, la etnicisation conlleva una explicación per se de lo que precisamente constituye el objeto de estudio. Ciertos usos hechos por investigadores, periodistas o incluso políticos, sugieren claramente que existe una forma patológica o pervertida de las relaciones sociales, fenómeno deplorable contra el cual es necesario luchar.

Cuestionando la popularidad de la expresión "etnicisation de las relaciones sociales" en Francia (y su ausencia en el exterior), este artículo examina hasta qué punto esta expresión ha contribuido a eludir las relaciones interétnicas actualmente presentes en Europa. Al mismo tiempo, el creciente uso de la palabra "etnicisation" podría permitir el reconocimiento de dimensiones no imaginadas de la etnicidad. 


\section{AUTEUR}

\section{HÉLĖNE BERTHELEU}

Maître de Conférence en Sociologie, Département de Sociologie, Laboratoire UMR CNRS 6173

CITERES. Université François Rabelais de Tours, 3 rue des Tanneurs, 37041 Tours Cedex 01

bertheleu@univ-tours.fr 